\title{
Biochanin A Suppresses Tumor Progression and PD-L1 Expression via Inhibiting ZEB1 Expression in Colorectal Cancer
}

\author{
Junying Xu, ${ }^{1}$ Xuejing Yang, ${ }^{1}$ Jiadong Pan, ${ }^{2}$ Honghong Fan, ${ }^{1}$ Jie Mei $\mathbb{C D}^{1},{ }^{1}$ and Dong Hua $\mathbb{D D}^{1}$ \\ ${ }^{1}$ Department of Oncology, The Affiliated Wuxi People's Hospital of Nanjing Medical University, Wuxi 214023, Jiangsu, China \\ ${ }^{2}$ Department of Gastroenterology, The Affiliated Wuxi People's Hospital of Nanjing Medical University, Wuxi 214023, \\ Jiangsu, China
}

Correspondence should be addressed to Dong Hua; wx89211@163.com

Received 9 December 2021; Revised 5 January 2022; Accepted 15 January 2022; Published 22 February 2022

Academic Editor: Jimei Wang

Copyright (C) 2022 Junying Xu et al. This is an open access article distributed under the Creative Commons Attribution License, which permits unrestricted use, distribution, and reproduction in any medium, provided the original work is properly cited.

\begin{abstract}
Objective. To investigate the regulatory effect of ZEB1 on PD-L1 expression and the pharmacodynamic effects of Biochanin A on the malignant biological behaviors of colorectal cancer (CRC). Methods. The correlation between epithelial-mesenchymal transition (EMT) score and features of the tumor microenvironment (TME) was investigated using the Cancer Genome Atlas (TCGA) dataset. The correlation between ZEB1 and PD-L1 expression was validated using immunohistochemistry (IHC) staining, and the regulatory effect of ZEB1 on PD-L1 expression was explored by in vitro assays. Moreover, the pharmacodynamic effects of Biochanin A on ZEB1 and PD-L1 expression, as well as malignant biological behaviors of CRC cells, were evaluated by in vitro and in vivo assays. Results. EMT score was positively correlated with a majority of immunostimulators, immune checkpoints, activities of antitumor immunity cycles, and infiltration levels of most immune cells in the TCGA dataset. In addition, ZEB1 was correlated with and positively regulated PD-L1 expression in CRC. Besides, Biochanin A, an inhibitor for the ZEB1/PD-L1 axis, notably inhibited ZEB1-mediated aggressiveness and PD-L1 expression of CRC cells. Moreover, Biochanin A also exerted a tumor-inhibitory role in vivo in the CRC mouse model. Conclusion. Overall, we found that ZEB1 is a main regulator of PD-L1 expression in CRC. In addition, we also identified Biochanin A as a novel inhibitor for the ZEB1/PD-L1 axis, which could inhibit tumor progression and immune escape.
\end{abstract}

\section{Introduction}

Colorectal cancer (CRC) is one of the most widespread digestive cancers, ranking third in both morbidity and mortality [1]. Based on the latest cancer statistics, approximately 149,550 cases of CRC will arise, and 52,980 CRCassociated deaths will occur in the United States in 2021 [1]. In recent years, the therapeutic strategies for CRC, including surgery, chemotherapy, radiotherapy, and immunotherapy, have been rapidly and largely developed. Thus, the 5-year overall survival (OS) rate for CRC patients has reached 65\% and even $90 \%$ in early-stage CRC patients $[2,3]$. Nonetheless, patients with advanced-stage CRC still face lethal clinical outcomes due to uncontrollable metastasis and other complications. Thus, it is urgent to explore the mechanisms underlying oncogenesis and search for more promising strategies for CRC.

Epithelial-mesenchymal transition (EMT) is a specific biological process in which epithelial cells transform into cells with a mesenchymal phenotype. EMT acts as a significant role in multiple physiological and pathological conditions, such as embryonic development, chronic inflammation, tissue reconstruction, tissue fibrosis, and malignant tumor progression [4]. Through the EMT process, epithelial cells lose cell polarity, the connection with basement membrane, and other epithelial features but obtain the mesenchymal features, such as high invasive capacity, antiapoptosis, and ability to degrade extracellular matrix [5]. Emerging studies reveal that EMT can significantly promote the progression and immune evasion of malignant tumors 
[6-8]. For example, kallikrein-related peptidase 8 (KLK8) promotes the growth and metastasis of CRC via activating the EMT process [9]. Therefore, the exploration of therapeutic strategies targeting the EMT process may be a critical breakthrough point to control the progression of CRC.

In the current study, we explored the correlated pattern of EMT-related gene expression and features of the tumor microenvironment (TME) utilizing the data from the Cancer Genome Atlas (TCGA) dataset. Next, the correlation and regulatory axis of ZEB1 and PD-L1 were investigated. Besides, we validated the tumor-suppressive role of Biochanin A in CRC in vitro and in vivo, which could suppress the ZEB1-mediated progression and PD-L1 expression. Overall, our findings uncover the critical role of ZEB1 in promoting tumor progression and identify a novel inhibitor Biochanin A to block the ZEB1/PD-L1 axis.

\section{Materials and Methods}

2.1. Public Data Acquisition and Bioinformatics Analysis. The normalized RNA sequencing (RNA-seq) for CRC from the TCGA dataset was obtained from the UCSC Xena portal (https://xenabrowser.net/datapages/). Next, the immunobiological correlations of EMT enrichment score in CRC were evaluated, which was calculated by single-sample gene set enrichment analysis (ssGSEA) [10] referring to a penal of EMT-associated genes belonging to gene sets "HALLMARKEPITHELIAL-MESENCHYMAL-TRANSITION" $\quad(n=200)$ in the Molecular Signatures Database (MSigDB) [11].

Given that the tumor tissues which were subjected to RNA-seq included tumor cells and other cells, such as immune cells, we assessed immunological characteristics of the TME for each patient from the TGCA dataset. First, the information of 122 immunomodulators and well-known effector genes of TIICs was also collected from previous studies [12]. Besides, ESTIMATE was used to estimate the Tumor Purity, ESTIMATE Score, Immune Score, and Stromal Score [13]. In addition, five independent algorithms, including TIMER [14], EPIC [15], MCP-counter [16], quanTIseq [17] and TISIDB [18], were utilized to compute the relative abundance of tumor-infiltrating immune cells (TIICs) comprehensively. Furthermore, considering that each stage of the cancer immune cycle plays a crucial role in reflecting the anticancer immune response and deciding the destiny of tumor cells, we next calculated the activities of each stage by ssGSEA according to the expression level of stage-specific signatures [10]. To investigate the immunobiological role of EMT in CRC, we divided the patients into the high and low EMT score groups at the 50\% cutoff criterion and then compared the difference of immunological features of TME between the high and low EMT subtypes.

2.2. Reagents and Antibodies. Biochanin A (Cat. B106472) was purchased from Aladdin (Shanghai, China). Ready-to-use PD-L1 antibody (rabbit mAb, Cat. GT2280) was purchased from GeneTech (Shanghai, China). Antibodies targeting ZEB1 (rabbit pAb, Cat. 21544-1-AP), PD-L1 (mouse mAb, Cat. 66248-1-Ig), E-cadherin (rabbit pAb, Cat. 20874-1-AP),
$\mathrm{N}$-cadherin (rabbit pAb, Cat. 22018-1-AP), and GAPDH (mouse mAb, Cat. 60004-1-Ig) were purchased from ProteinTech (Wuhan, China).

2.3. Tissue Microarray. The CRC tissue microarray (TMA) section (Cat. HColA160Bc01) was obtained from Outdo BioTech (Shanghai, China). This TMA contained 80 tumor and their paired paratumor samples. The relevant medical record of clinic-pathological features for each sample was obtained from Outdo BioTech. Ethical approval for the study of the TMA was granted by the Clinical Research Ethics Committee, Outdo BioTech (Shanghai, China).

2.4. Immunohistochemistry. Immunohistochemistry (IHC) staining was performed on the section according to the standard steps. Briefly, the TMA was deparaffinized at $55^{\circ} \mathrm{C}$ for $30 \mathrm{~min}$ and then washed with xylene for three $5 \mathrm{~min}$. The section was rehydrated by successive washes in $100 \%, 90 \%$, and $70 \%$ graded ethanol. Hydrogen peroxidase $(0.3 \%$, ZSGB-Bio, Beijing, China) was used to block endogenous peroxidase activity for $20 \mathrm{~min}$. Then, the section was retrieved by EDTA. The primary antibodies were as follows: anti-ZEB1 (1:4000 dilution, Cat. 21544-1-AP, ProteinTech) and anti-PD-L1 (Ready-to-use, Cat. GT2280, GeneTech). The immunostained section was scanned using Aperio Digital Pathology Slide Scanner. The semiquantitative assessment was conducted according to the 12-point criteria as described previously, and the semiquantitative result was defined as immunoreactivity score (IRS) [19].

2.5. Cell Culture and Transfection. Human CRC cell lines HCT116 and SW620 were purchased from KeyGEN (Nanjing, China). HCT116 cells were maintained in McCoy's 5A media supplemented with $10 \%$ fetal bovine serum (FBS) at $37^{\circ} \mathrm{C}$ with $5 \% \mathrm{CO}_{2}$. SW620 cells were cultured in Leibovitz's L-15 media supplemented with $10 \%$ FBS at $37^{\circ} \mathrm{C}$ with $5 \%$ $\mathrm{CO}_{2}$. All experiments were performed with mycoplasma-free cells. The HCT116 and SW620 cell lines have recently been authenticated using short tandem repeat profiling.

For knockdown or upregulation of ZEB1 expression, CRC cells were cultured in 6-well plates to $60-80 \%$ confluence and transfected with small interfering RNA (siRNA) and overexpression vector for ZEB1, which is synthesized in KeyGEN, using Lipofectamine 3000 Reagent (Cat. L3000015, Invitrogen, CA, USA) according to the manufacturer's instructions. The sequence of ZEB1 siRNAs were as follows: siRNA-1: AGGAAGAGGAGGAGGAUAATT, siRNA-2: ACACAUAAGCAGUAAGAAATT, and siRNA-3: GGCAAAAGAUAGAGAAUAATT. After 48 hours, the total RNA and protein of CRC cells were extracted and submitted for quantitative real-time PCR (qRT-PCR) and Western blotting analysis to check the efficiency of ZEB1 knockdown and overexpression.

2.6. Quantitative Real-Time PCR. The total RNA of HCT116 and SW620 cells was collected by TRIzol reagent (Cat. 15596026, Invitrogen, CA, US). The primers for ZEB1, PD- 
L1, and GAPDH mRNA reverse transcription were synthesized in KeyGEN (Nanjing, China). qRT-PCR was conducted using the One-Step TB GreenTM PrimeScriptTM RT-PCR Kit II (SYBR Green) (Cat. RR086 B, TaKaRa, Kyoto, Japan).

Primers used for gene amplification were as follows: ZEB1: (forward) CGCTTCTCACACTCTGGGTCTT and (reverse) CCTCTTCCTGCTCTGTGCTGTC; PD-L1: (forward) GCCGAAGTCATCTGGACAAGC and (reverse) TG ATTCTCAGTGTGCTGGTCAC; GAPDH: (forward) AGA TCATCAGCAATGCCTCCT and (reverse) TGAGTCCT TCCACGATACCAA.

2.7. Western Blotting Analysis. CRC cells were plated in a 6well plate for transfection. After 48 hours, the total protein of HCT116 and SW620 cells was harvested using lysis buffer. Then, SDS-PAGE and Western blotting analyses were conducted as standard protocols. The primary antibodies used were as follows: ZEB1 (1:1000 dilution, Cat. 21544-1AP, ProteinTech), E-cadherin $(1: 1000$ dilution, Cat. 208741-AP, ProteinTech), N-cadherin (1:1000 dilution, Cat. 22018-1-AP, ProteinTech), PD-L1 (1:2000 dilution, Cat. 66248-1-Ig, ProteinTech), and GAPDH (1:5000 dilution, Cat. 60004-1-Ig, ProteinTech). Expression levels of these proteins were standardized to GAPDH for each specimen.

2.8. Immunofluorescence. The subcellular locations of proteins were assessed using immunofluorescence assay according to standardized protocols [20]. The primary antibodies used were as follows: ZEB1 (1:100 dilution, Cat. 21544-1-AP, ProteinTech), E-cadherin (1:100 dilution, Cat. 20874-1-AP, ProteinTech), N-cadherin (1:100 dilution, Cat. 22018-1-AP, ProteinTech), and PD-L1 (1:100 dilution, Cat. 66248-1-Ig, ProteinTech). The stained cells were observed under a fluorescence microscope (FV3000, Olympus).

2.9. CCK-8 Assay. HCT116 and SW620 cells were digested using $0.25 \%$ trypsin for $1 \mathrm{~min}$ and resuspended with DMEM media containing $10 \%$ FBS. Suspended cells were seeded on a 96-well plate with the cell density adjusted to $5 \times 10^{4}$ cells/ $\mathrm{ml}(100 \mu \mathrm{l} /$ well $)$ and fostered at $37^{\circ} \mathrm{C}$ in a constant-temperature incubator with $5 \% \mathrm{CO}_{2}$ for 48 hours. To each well, $10 \mu \mathrm{l}$ CCK- 8 was added, after which the plate was put in the incubator for 1 hour. The OD value of each well was measured at $450 \mathrm{~nm}$ by a microplate reader.

2.10. Wound Healing Assay. For cell migration assays, HCT116 and SW620 cells were seeded in 6-well plates (Costar, Corning, NY) and cultured to $80 \%$ confluence. The monolayers of cells were wounded by removing the cultureinsert and rinsed with PBS to remove cell debris. After the $24 \mathrm{~h}$ migration, cells were stained with $0.2 \%$ crystal violet for $20 \mathrm{~min}$ at room temperature. The images were acquired at times $0 \mathrm{~h}$ and $24 \mathrm{~h}$ after migration using a microscope (magnification: 100×, OLYMPUS IX51). The migratory distance was calculated by the minus of the edge of the wound closure between $0 \mathrm{~h}$ and $24 \mathrm{~h}$.
2.11. Boyden Chamber Assay. For cell invasion assays, $1 \times 10^{5}$ cells in serum-free medium supplemented with $5 \mathrm{mg} /$ $\mathrm{mL}$ BSA were inoculated to the upper sides of the modified Boyden chamber (8.0- $\mu \mathrm{m}$, Cat. 3422, Corning, NY, US). The polycarbonate membranes of Boyden chambers were coated with Matrigel (BD Biosciences, NJ, US). After 24 hours, the invasive cells on the lower sides of Boyden chambers were fixed and stained with $0.2 \%$ crystal violet for $20 \mathrm{~min}$ at room temperature. The pictures of stained cells were captured by a microscope (magnification: 200×, OLYMPUS IX51).

2.12. In Vivo Tumorigenesis Analysis. C57BL/6 mice (4-5 weeks old) were purchased from Shanghai SLAC Laboratory Animal Co., Ltd. The mice were raised in SPF-grade experimental animal centers and provided with free access to food and water. To establish the syngeneic mouse model, PD-L1 positive MC38 mouse tumor cells [21, 22] maintained in DMEM media supplemented with 10\% FBS were subcutaneously injected into the flanks of these female mice $\left(1 \times 10^{7}\right.$ cells). Tumors were monitored and regularly measured with calipers every two to three days. When tumors reached about $100 \mathrm{~mm}^{3}$ in volume, mice were randomized into two different groups $(n=6)$ : vehicle group and Biochanin A-treated group. Biochanin A was dissolved in PBS and administered to mice through intraperitoneal injection at $50 \mathrm{mg} / \mathrm{kg}$ daily. On day 21, the mice were anesthetized with a $0.5 \%$ sodium pentobarbital solution to remove the tumor and photographed and weighted, and the tissues were submitted for IHC staining. All experiments were approved by the Laboratory Animal Ethics Committee at the Affiliated Wuxi People's Hospital of Nanjing Medical University. Mouse tumor tissues were manufactured into $4 \mathrm{~mm}$ thick formalinfixed and paraffin-embedded sections. IHC for these sections was subsequently conducted. The primary antibodies used in the research were as follows: anti-ZEB1 (1:500 dilution, Cat. 21544-1-AP, ProteinTech) and anti-PD-L1 (1:100 dilution, Cat. 66248-1-Ig, ProteinTech). Semiquantitative evaluation criteria were as described previously.

2.13. Statistical Analysis. All statistical analyses were conducted using SPSS 26.0 software (Chicago, IL). Most of the data were analyzed by Student's $t$-test or Mann-Whitney test. All data are presented as means \pm SDs. Correlation analysis between two variables was examined by Pearson's test. Two-sided $P$ value $\leq 0.05$ was considered statistically significant labeled with ${ }^{*} p<0.05 ;{ }^{* *} p<0.01 ;{ }^{* * *} p<0.01$.

\section{Results}

3.1. EMT Was Associated with Features of TME in CRC. Previous research revealed that EMT was correlated with PD-L1 expression in clinical lung cancer cohorts [23]. Thus, we first investigated the immunological role of EMT in CRC in this research. A majority of immunostimulators, major histocompatibility complex (MHC) molecules, chemokines, and chemokine receptors were highly expressed in the high EMT group (Figure 1(a)). Tumor Purity, ESTIMATE Score, Immune Score, and Stromal Score were calculated by the 


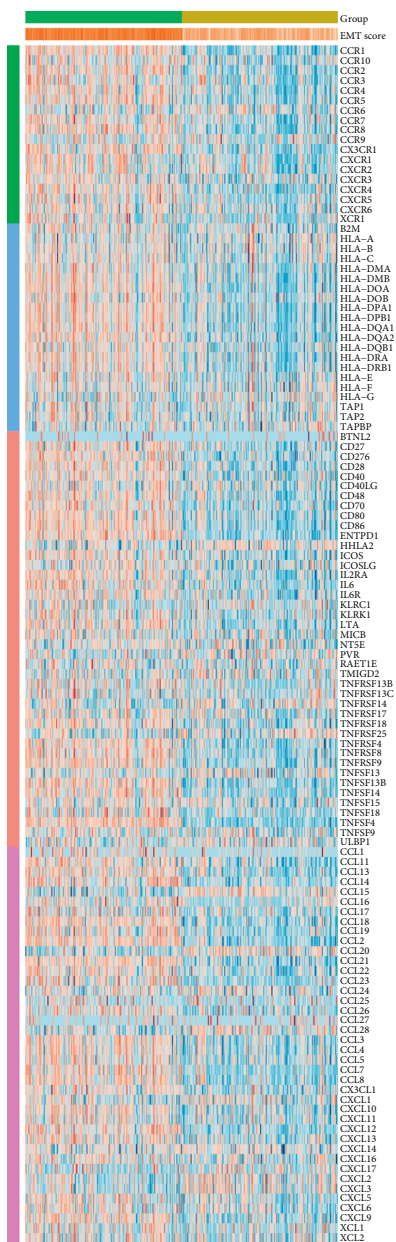

(a)

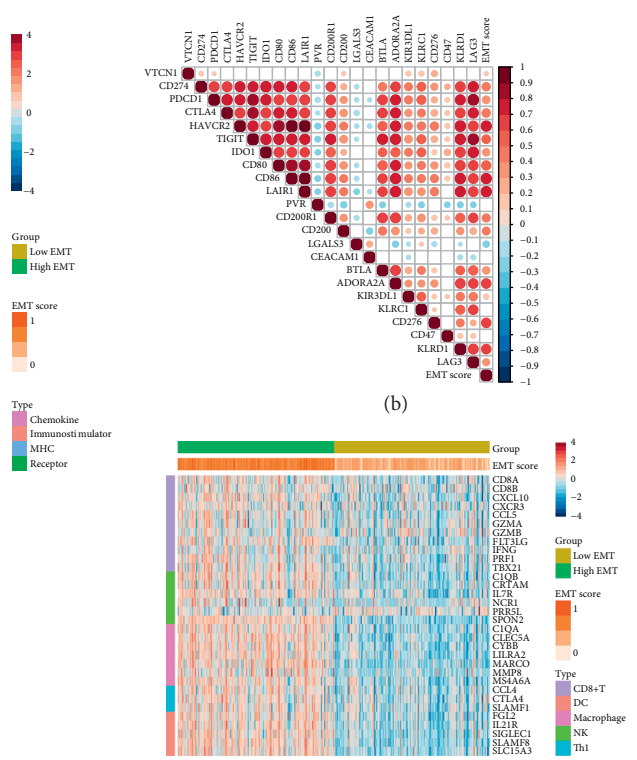

(c)

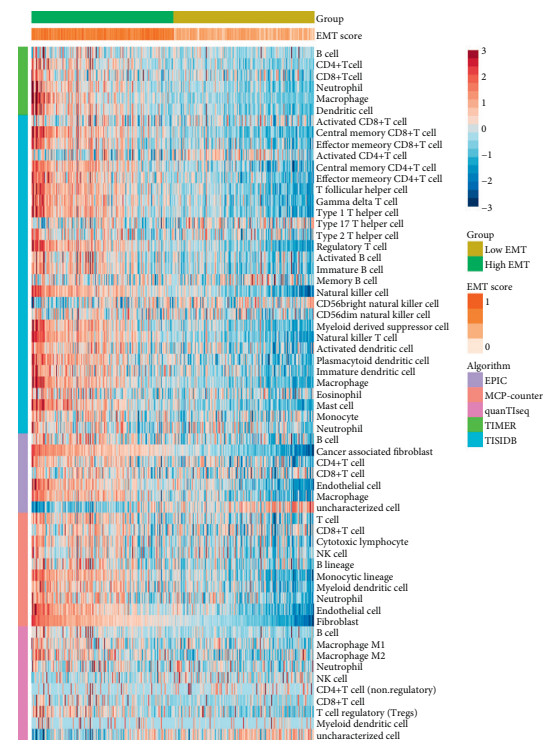

(d)

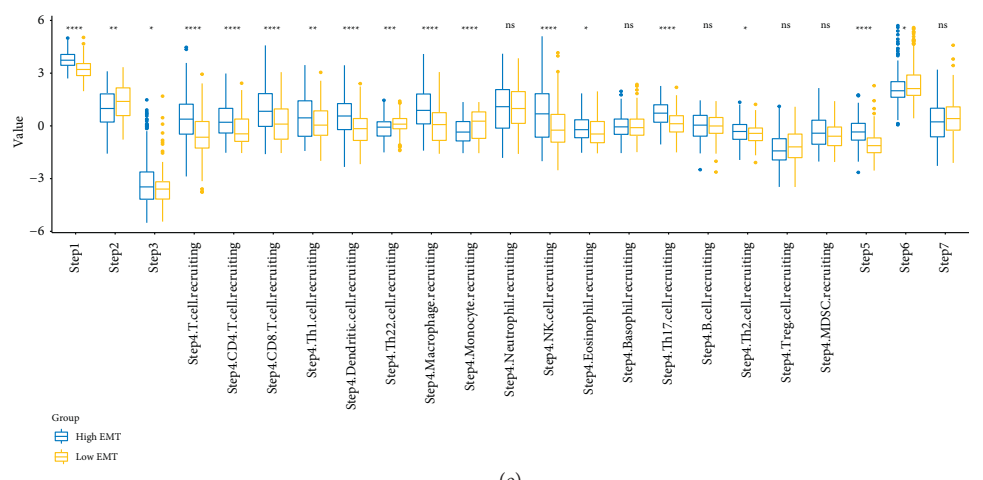

Figure 1: EMT was related to the features of TME in CRC. (a) Expression levels of 122 immunomodulators between the high and low EMT groups. (b) Correlation between EMT score and inhibitory immune checkpoints. The color reveals the Pearson correlation coefficient. (c) Expression levels of gene markers of immune cells between the high and low EMTgroups. (d) The difference in TIICs levels between high and low EMT groups calculated by five algorithms. (e) Differences in the various steps of the cancer immunity cycle between the high and low EMT groups.

ESTIMATE method. The results showed that the EMT score was positively correlated with ESTIMATE Score, Immune Score, and Stromal Score but negatively correlated with Tumor Purity (Figures S1(a)-S1(d)). EMT score was revealed to be positively related to a majority of immune checkpoints, including PD-L1, PD-1, and CTLA-4 (Figure 1(b)). In addition, the EMT score was positively related to most gene markers of immune cells (Figure 1(c)). Next, we also estimated the infiltration level of TIICs based on five independent algorithms. EMT score was positively related to the infiltration levels of most immune cells (Figure 1(d)). Furthermore, the activities of most steps in antitumor immunity cycles were enhanced in the high EMT group (Figure 1(e)). Overall, these findings revealed that EMT was significantly related to the inflammatory TME in CRC.

3.2. ZEB1 Was Positively Related to and Regulated PD-L1 Expression in CRC. Among the regulators of the EMT process, ZEB1 has been proved to be a critical regulator for
PD-L1 expression in lung cancer [23], breast cancer [24], gastric cancer [25], and diffuse large B cell lymphoma [26]. Thus, we investigated the correlation between ZEB1 and PD-L1 in CRC. First, ZEB1 was upregulated in CRC tissues compared with paratumor tissues (Figures 2 (a) and 2(b)) and positively correlated with tumor stages, including $\mathrm{N}$ stage, $M$ stage, and TNM clinical stage (Figures 2(c)-2(f)). In addition, ZEB1 was positively correlated with PD-L1 expression revealed by IHC staining (Figures 2(g)-2(h)). Next, we investigated whether ZEB1 regulated PD-L1 expression in CRC cells. The efficiency of siRNAs for ZEB1 silencing was checked, and the results showed that siRNA-2 (ACACAUAAGCAGUAAGAAATT) had a satisfactory silencing efficiency in both HCT-116 and SW620 cells (Figures S2(a) and S2(b)). In addition, the overexpression efficiency of the ZEB1 vector was also validated (Figures S2(c)-S2(d)). Inhibition of ZEB1 significantly downregulated PD-L1 expression (Figures 3(a)-3(d)), but ZEB1 overexpression notably upregulated PD-L1 in both 


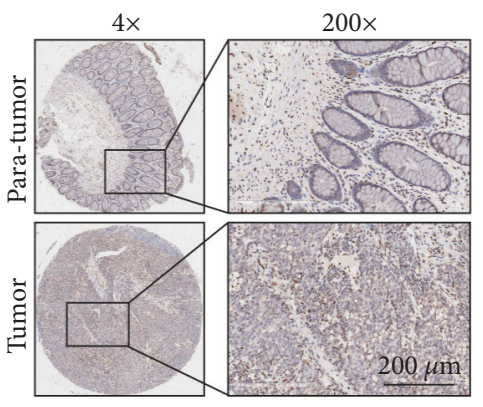

(a)

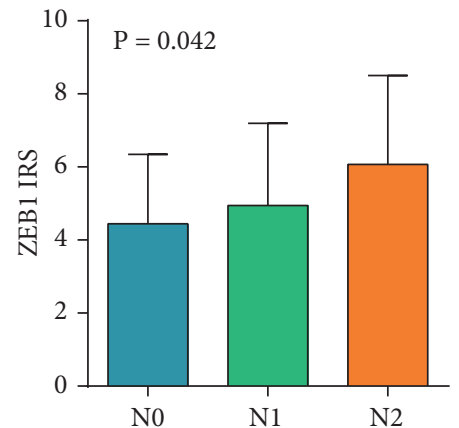

(d)

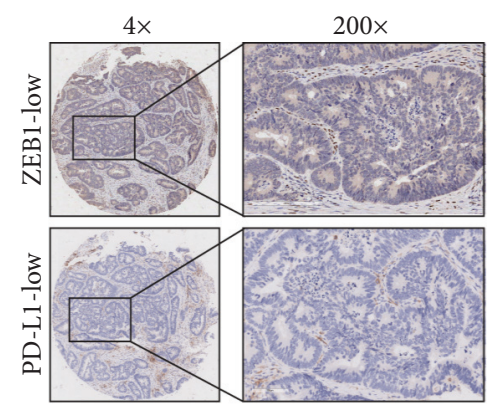

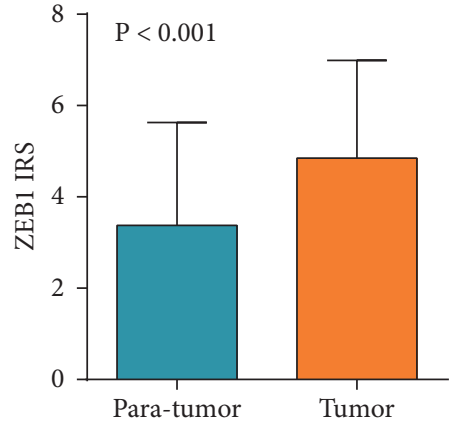

(b)

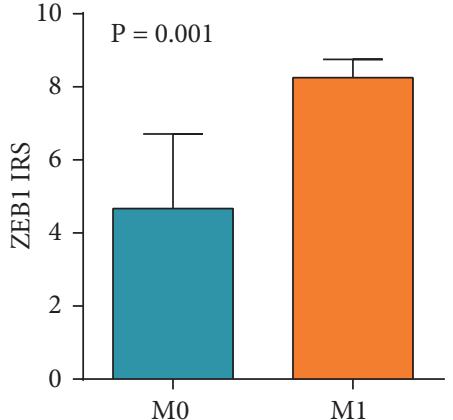

(e)

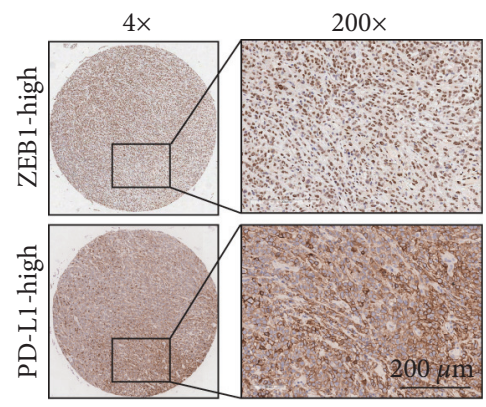

(g)

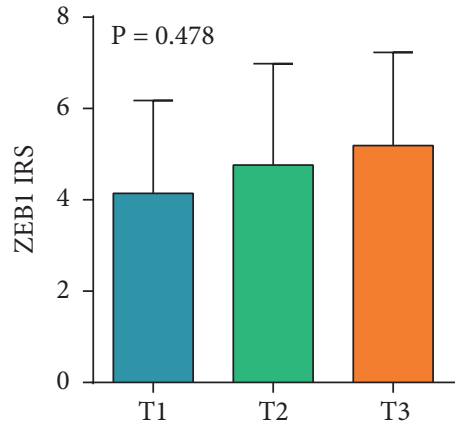

(c)

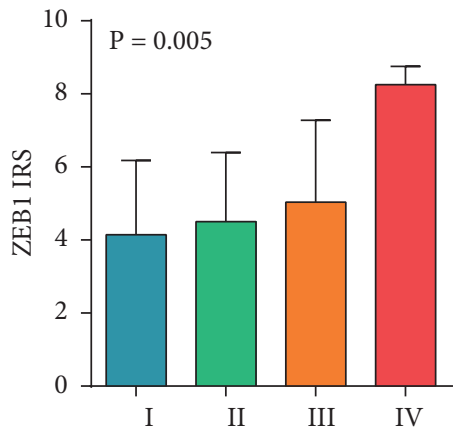

(f)

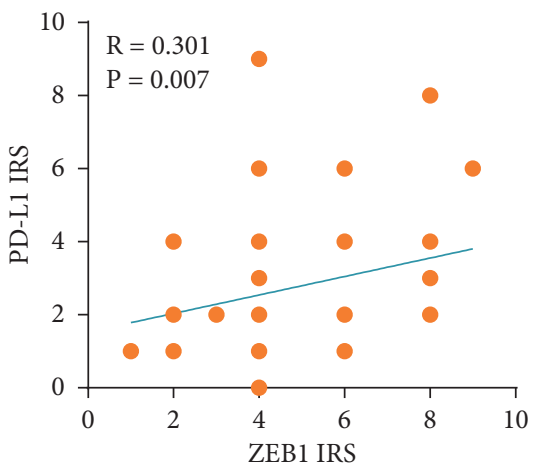

(h)

Figure 2: Expression of ZEB1 in CRC and its correlation with PD-L1 expression. (a) Representative images uncovering ZEB1 expression in paratumor and tumor tissues using anti-ZEB1 staining. (b) Semiquantitative analysis of ZEB1 in paratumor and tumor tissues. (c) Semiquantitative analysis of ZEB1 in CRC tissues with different T stages. (d) Semiquantitative analysis of ZEB1 in CRC tissues with different $\mathrm{N}$ stages. (e) Semiquantitative analysis of ZEB1 in CRC tissues with different M stages. (f) Semiquantitative analysis of ZEB1 in CRC tissues with different TNM clinical stages. (g) Representative images uncovering low and high ZEB1 and PD-L1 expressions in CRC tissues using anti-ZEB1 and anti-PD-L1 staining. (h) Correlation between ZEB1 and PD-L1 expressions in CRC tissues.

HCT-116 and SW620 cells (Figures 3(e)-3(h)). Taken together, these findings suggested that ZEB1 might be a critical regulator for PD-L1 expression in CRC as well.

\subsection{Biochanin A Suppressed ZEB1 Expression and the EMT} Process in CRCCells. Biochanin A was reported as an inhibitor for the EMT process [27]. Therefore, we checked the pharmacological effects of Biochanin A on ZEB1 expression and the EMT process in CRC cells. Low doses $(20 \mu \mathrm{M}$ and $60 \mu \mathrm{M})$ of Biochanin A slightly upregulated but a high dose $(100 \mu \mathrm{M})$ significantly inhibited ZEB1 expression in HCT116 cells
(Figures 4(a) and 4(b)). In addition, a high dose of Biochanin A also downregulated ZEB1 expression in SW620 cells (Figures 4(a) and 4(b)). Next, immunofluorescence assay also proved that a high dose of Biochanin A downregulated ZEB1 expression, especially nuclear ZEB1 expression in CRC cells (Figures 4(c) and 4(d)). We also checked the effects of Biochanin A on the EMT process. The results exhibited that Biochanin A transformed CRC cells into epithelial phenotype and upregulated $\mathrm{E}$-cadherin but inhibited $\mathrm{N}$-cadherin expression in CRC cells (Figures 4(e)-4(h)). Collectively, all evidence supported that Biochanin A inhibited ZEB1 expression and blocked the EMT process in CRC. 

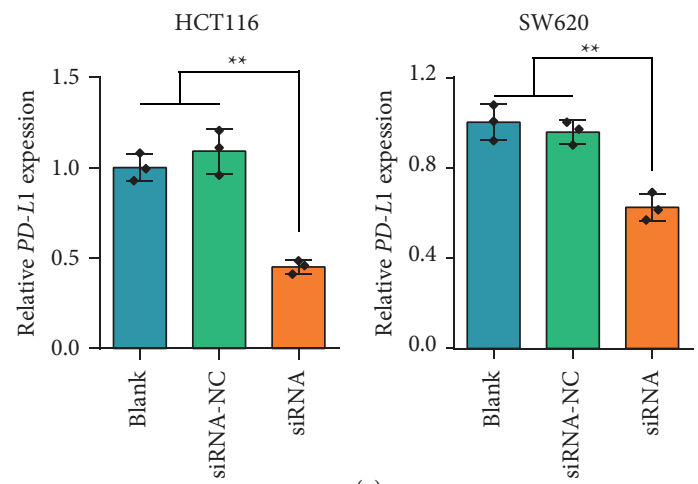

(a)
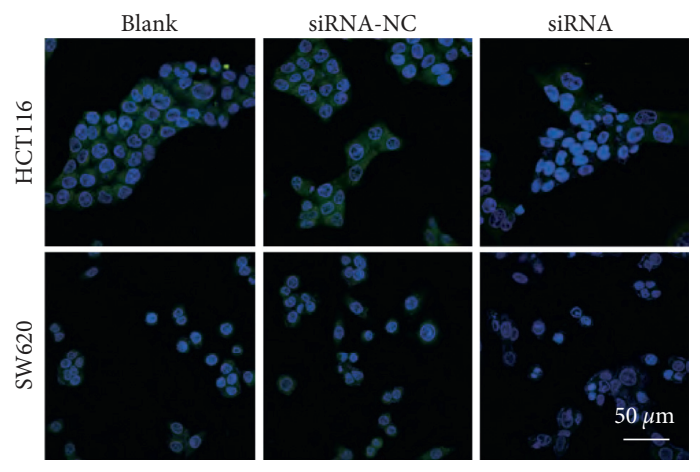

(c)
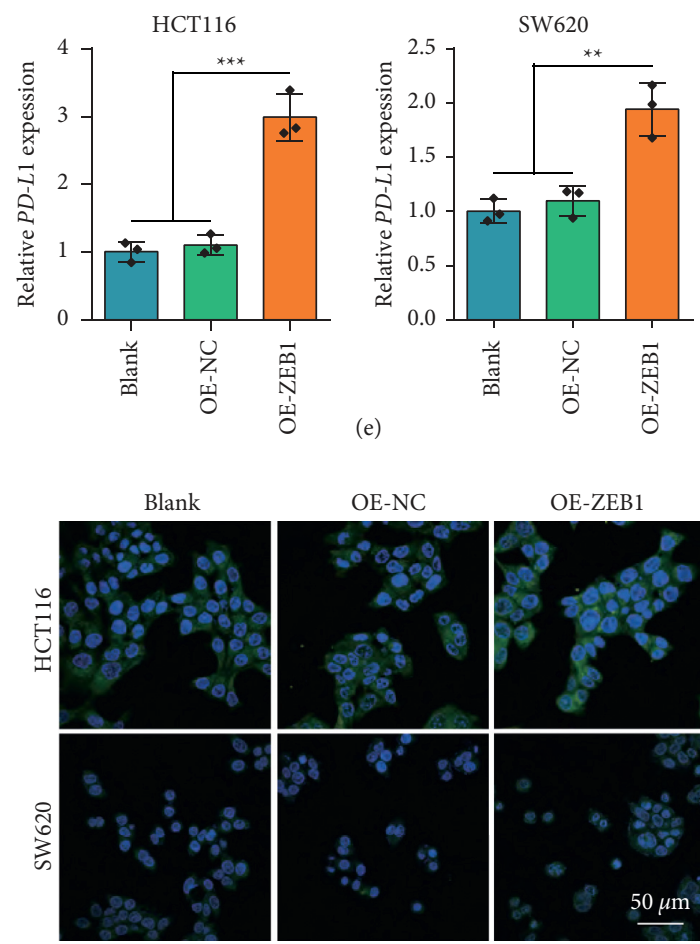

\begin{abstract}
OE-NC
\end{abstract}

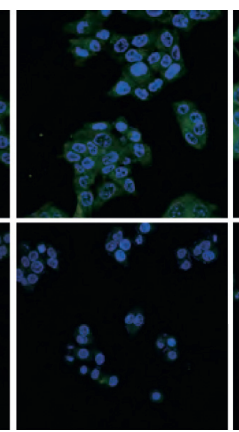

(g)

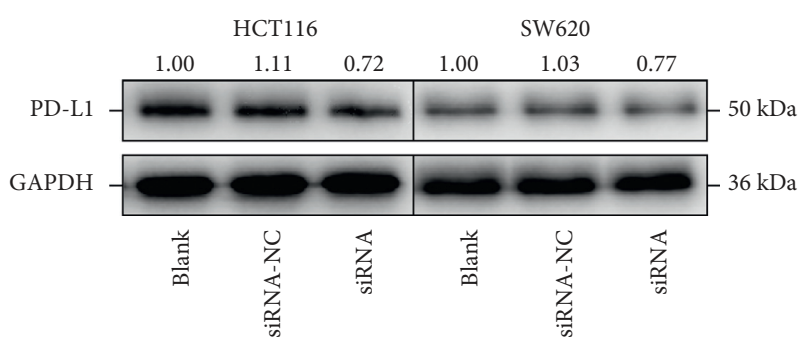

(b)
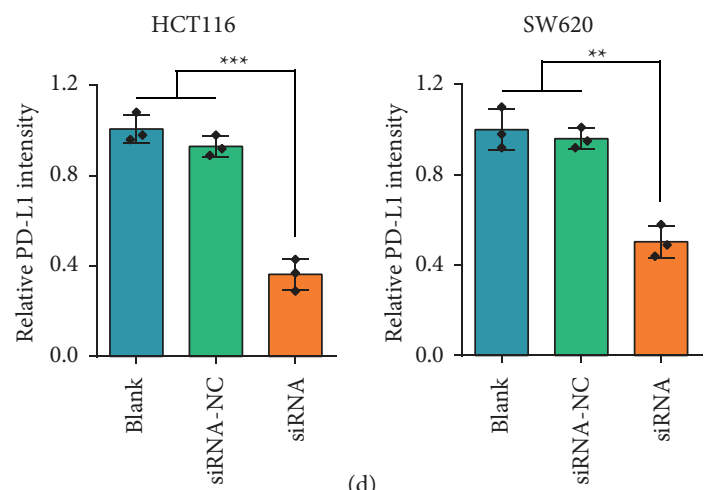

(d)

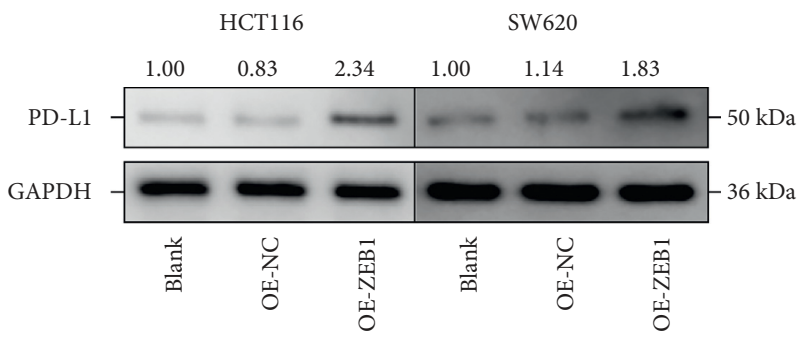

(f)
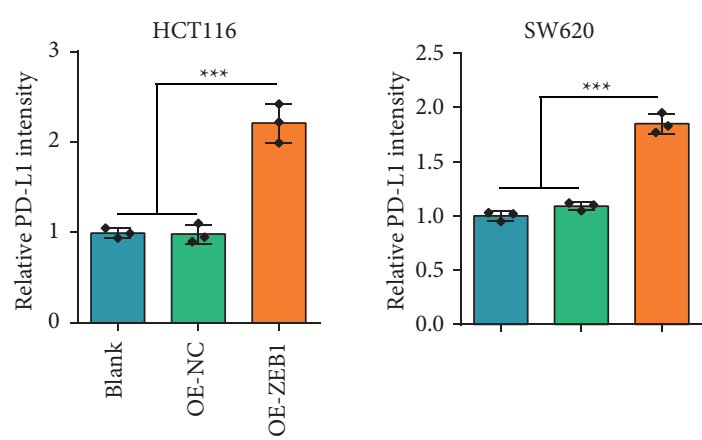

(h)

FIGURE 3: The regulatory effect of ZEB1 on PD-L1 expression in CRC. (A, B) Transcriptional and protein expressions of ZEB1 in CRC cells with ZEB1 silencing were examined by qPCR and Western blotting. (C, D) Protein expression of PD-L1 in CRC cells with ZEB1 silencing was examined by immunofluorescence. (E, F) Transcriptional and protein expressions of ZEB1 in CRC cells with ZEB1 overexpression were examined by qPCR and Western blotting. (G, H) Protein expression of PD-L1 in CRC cells with ZEB1 overexpression was examined by immunofluorescence. 

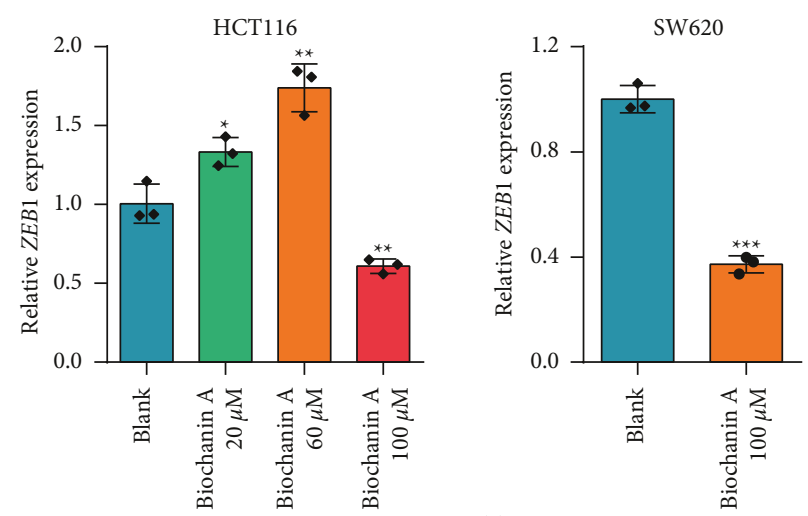

(a)

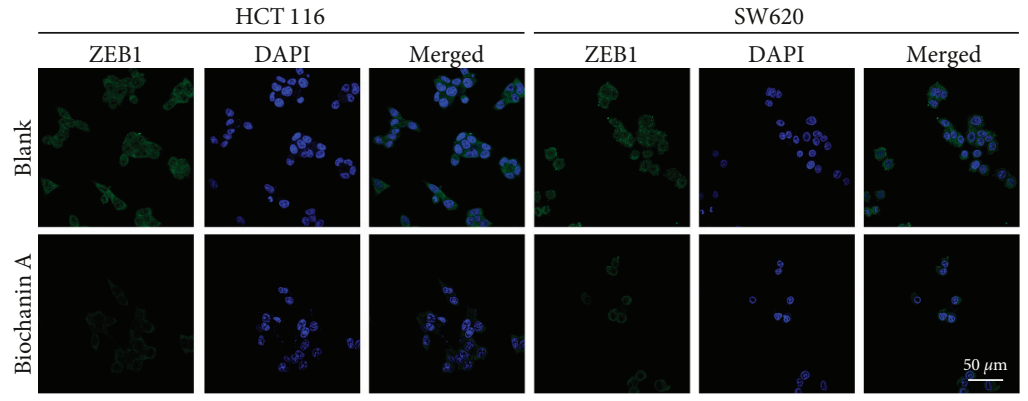

(c)

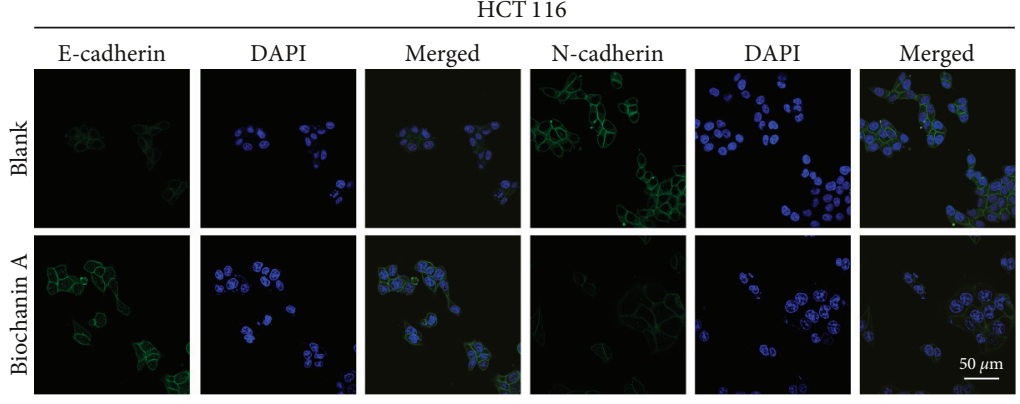

(e)

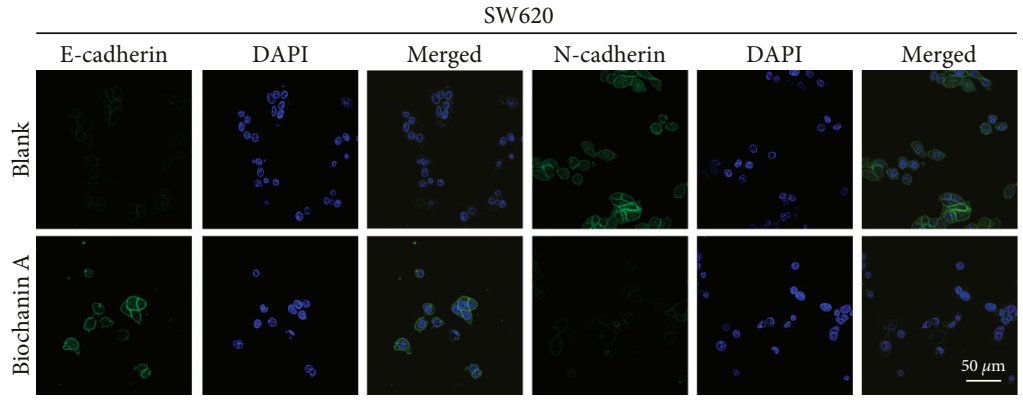

(g)

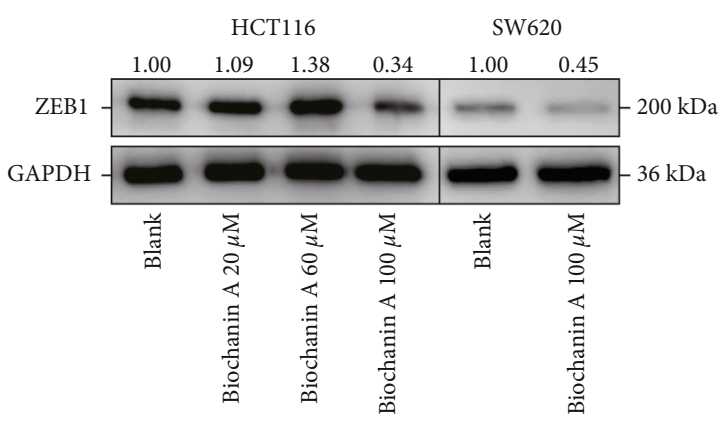

(b)
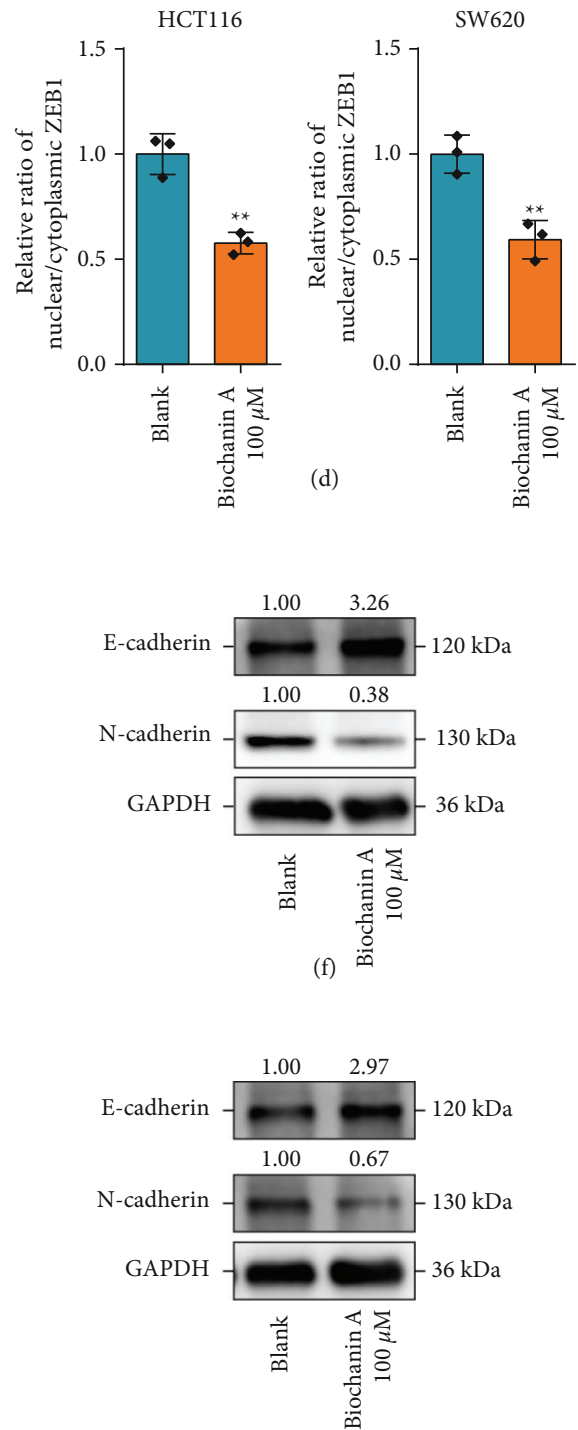

(h)

Figure 4: The effect of Biochanin A on ZEB1 expression and EMT in CRC. (A, B) Transcriptional and protein expressions of ZEB1 in CRC cells treated with Biochanin A were examined by qPCR and Western blotting. (C, D) Protein expression of ZEB1 in CRC cells treated with Biochanin A was examined by immunofluorescence. (E, G) Protein expression of E-cadherin and N-cadherin in CRC cells treated with Biochanin A was examined by immunofluorescence. (F, H) Protein expression of E-cadherin and N-cadherin in CRC cells treated with Biochanin A was examined by Western blotting. 

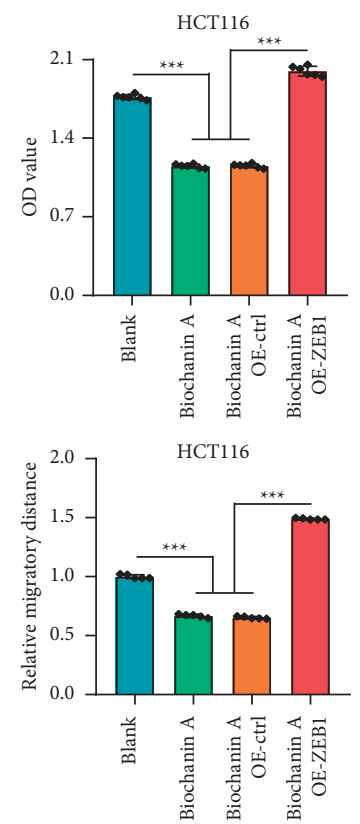

(a)
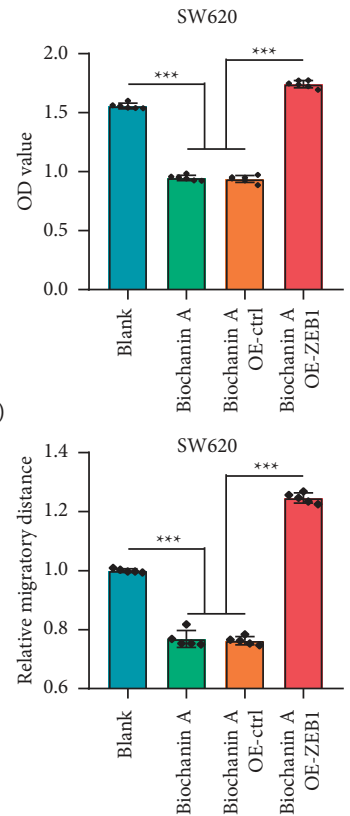

(c)

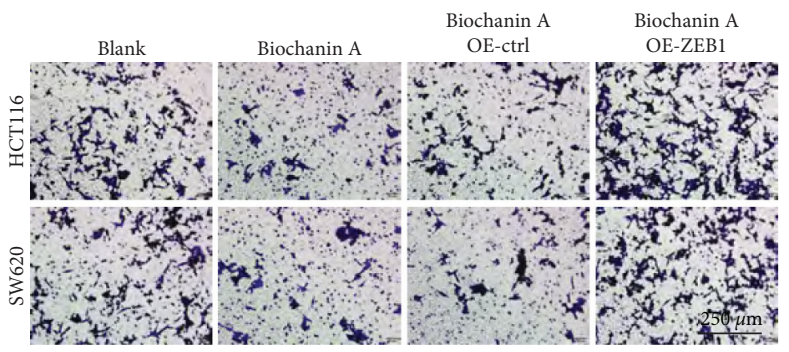

(d)

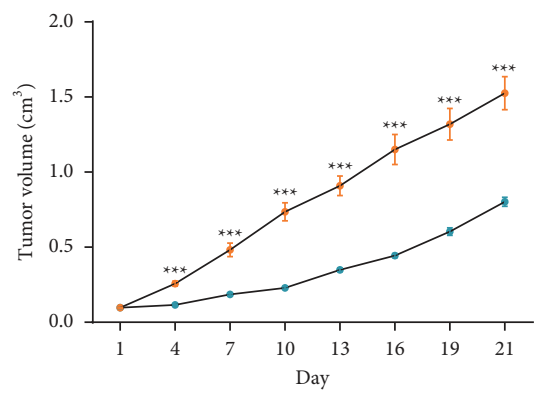

$\rightarrow$ Vehicle

$\rightarrow$ Biochanin A
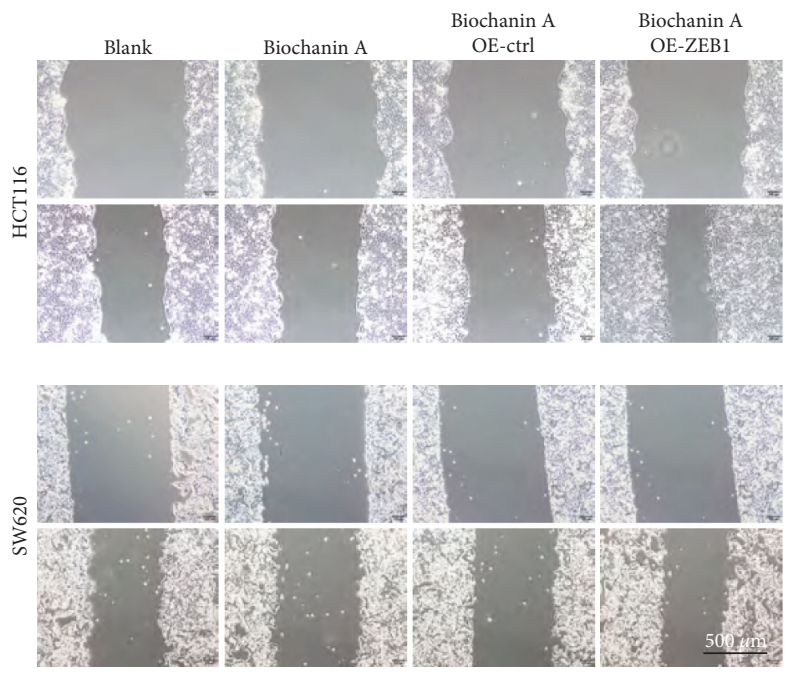

(b)
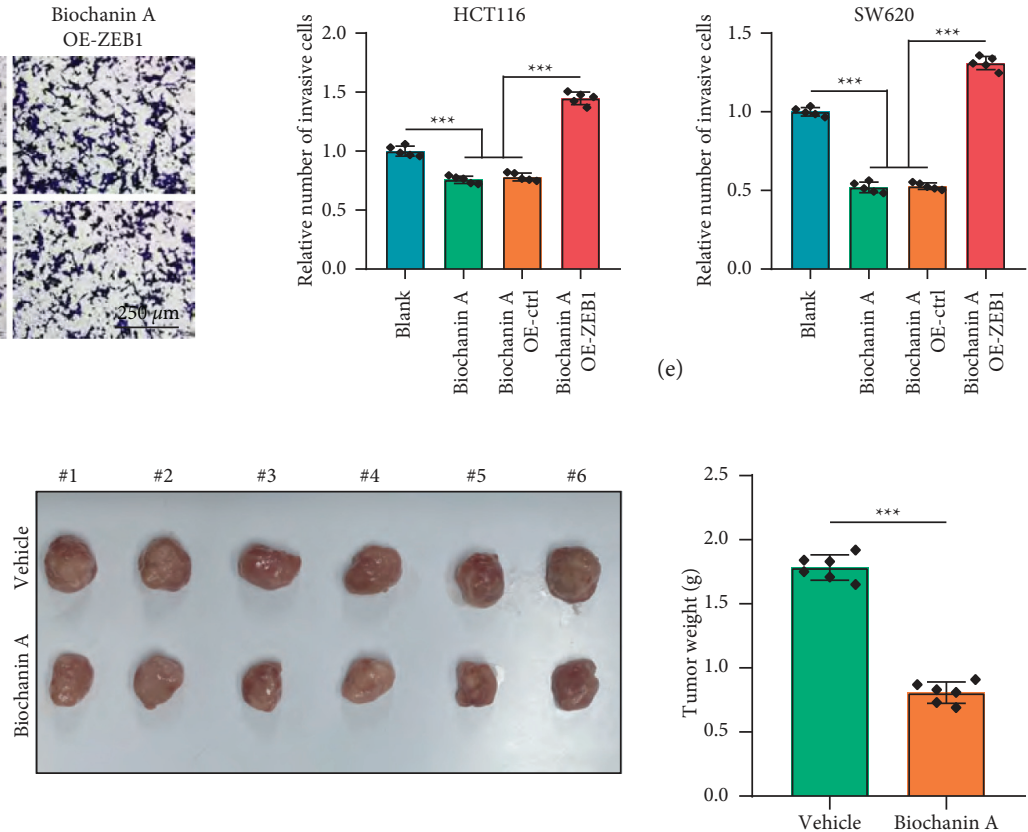

(g)

(h)

FIgURE 5: Tumor-suppressive role of Biochanin A in CRC in vitro and in vivo. (a) The proliferative capacity of control and Biochanin A-treated CRC cells was examined by CCK- 8 assay. (b) The migratory capacity of control and Biochanin A-treated CRC cells was examined by wound healing assay. (c) Quantitative analysis of cell migratory capacity. (d) The invasive capacity of control and Biochanin A-treated CRC cells was examined by Boyden chamber assay. (e) Quantitative analysis of cell invasive capacity. (f) Tumor volume of the mouse models, which was monitored every three days. (g) Isolated tumor images from these two groups on day 21 after the first treatment. (h) The tumor weight of these two groups; mice were sacrificed on day 21 after the first treatment.

3.4. Biochanin A Suppressed ZEB1-Mediated Aggressiveness of CRC Cells. We next assessed the tumor-suppressive role of Biochanin A in CRC cells. Compared with the control cells, Biochanin A-treated HCT-116 and SW620 cells exhibited attenuated proliferative capacity (Figure 5(a)). In addition, Biochanin A treatment significantly inhibited the migratory and invasive capacities of CRC cells (Figures 5(b)5(e)). However, ZEB1 overexpression rescued Biochanin A-induced inhibition of proliferation, migration, and invasion (Figures 5(a)-5(e)). Moreover, the antitumor pharmacodynamic of Biochanin A in vivo was also evaluated. The results showed that Biochanin A notably inhibited tumor 

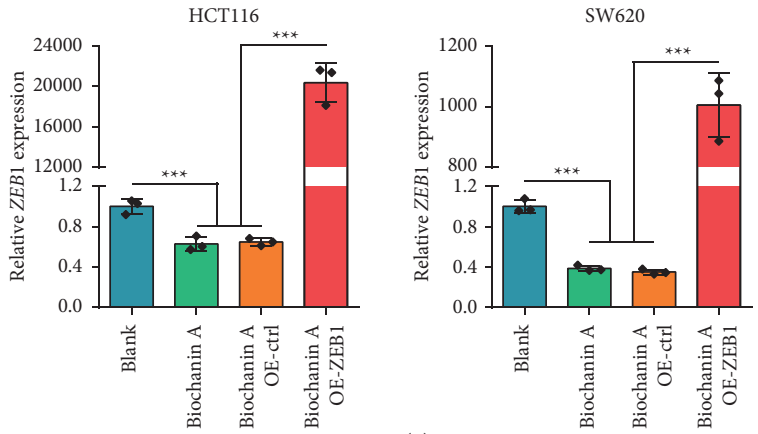

(a)
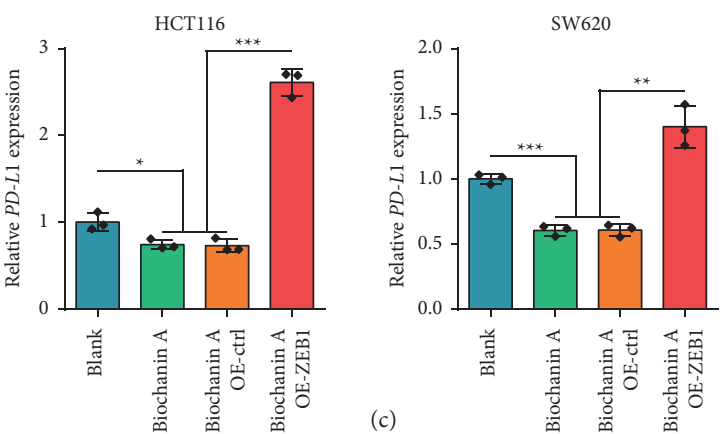

Biochanin A

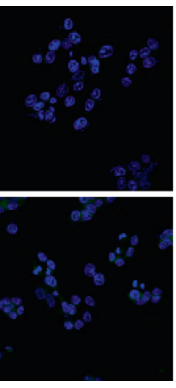

(e)

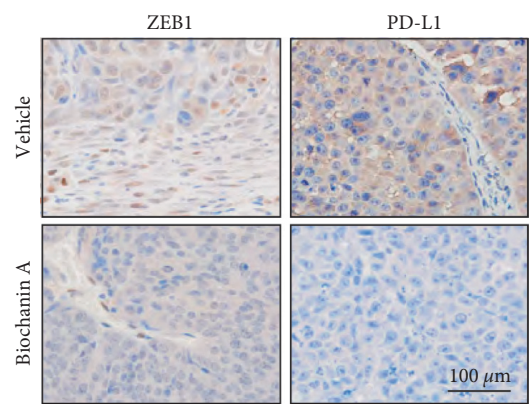

(g)
Biochanin A

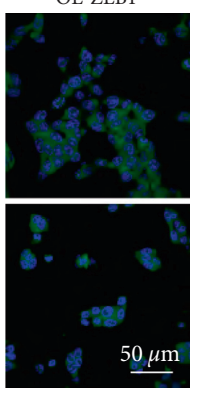

$50 \underline{\mu \mathrm{m}}$

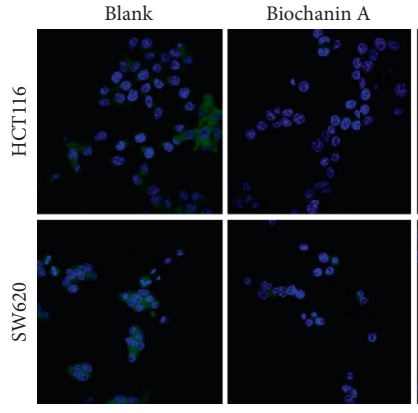

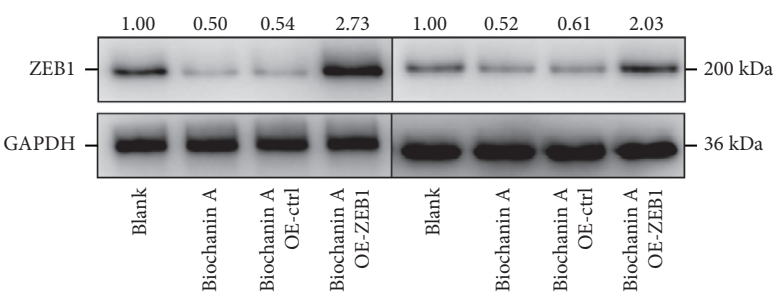

(b)

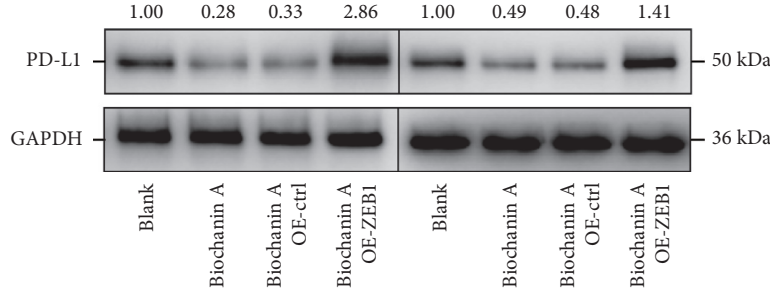

(d)

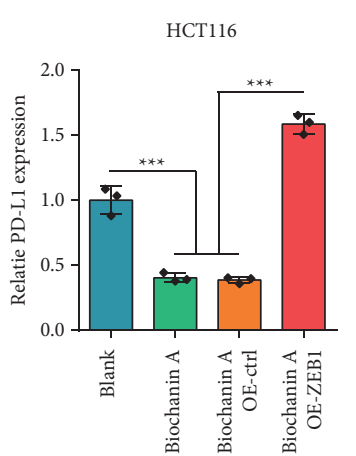

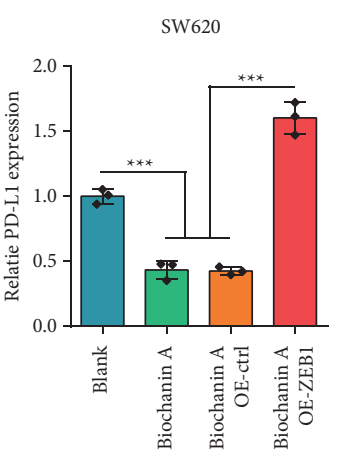

(f)

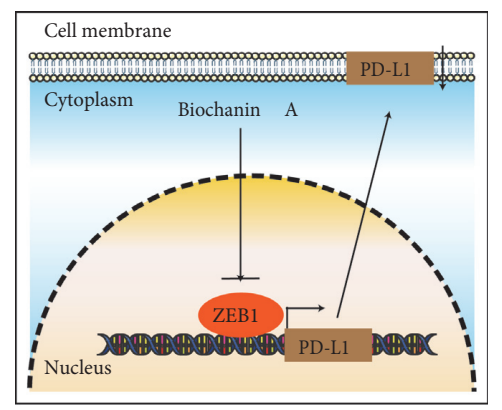

(i)

Figure 6: The effect of Biochanin A on PD-L1 expression in CRC. (A, B) Transcriptional and protein expressions of ZEB1 in CRC cells treated with Biochanin A or transfected with ZEB1 vector were examined by qPCR and Western blotting. (C, D) Transcriptional and protein expressions of PD-L1 in CRC cells treated with Biochanin A or transfected with ZEB1 vector were examined by qPCR and Western blotting. (E, F) Protein expression of PD-L1 in CRC cells treated with Biochanin A or transfected with ZEB1 vector was examined by immunofluorescence. $(G)$ Representative images uncovering ZEB1 and PD-L1 expression in vehicle and Biochanin A treated groups. (H) Semiquantitative analysis of ZEB1 and PD-L1 in mouse tumor tissues. (I) Schematic diagram of the mechanism underlying Biochanin A-induced ZEB1-mediated PD-L1 downregulation. 
growth of MC-38 mouse CRC cells in vivo (Figures 5(f)5(h)). Overall, Biochanin A significantly suppressed CRC progression in vitro and in vivo via downregulating ZEB1 expression, which could be used as a novel antitumor drug.

3.5. Biochanin A Suppressed ZEB1-Mediated PD-L1 Expression. Given the crucial regulatory role of ZEB1 in PDL1 expression, we also checked the effect of Biochanin A on PD-L1 expression. Predictably, Biochanin A inhibited ZEB1 and PD-L1 overexpression, and overexpression of ZEB1 largely rescued Biochanin A-induced PD-L1 downregulation (Figures $6(\mathrm{a})-6(\mathrm{~d})$ ). In addition, the result was also validated by immunofluorescence assay (Figures 6(e)6(f)). Furthermore, IHC analysis of mouse tumor tissues revealed that Biochanin A suppressed ZEB1 and PD-L1 expression in vivo as well (Figures $6(\mathrm{~g})$ and $6(\mathrm{~h})$ ). To sum up, Biochanin A was an antitumor immunomodulator via inhibiting ZEB1-mediated PD-L1 expression and thus increased the immune cell infiltration (Figure 6(i)).

\section{Discussion}

As one of the significant features of malignant tumors, immune escape plays a significant role in the oncogenesis, progression, and therapeutic resistance of malignant tumors [28]. Immunotherapeutic drugs represented by PD-1/ PD-L1 inhibitors have been widely used in multiple malignant diseases [29-32]. PD-L1 could inhibit the proliferation of $\mathrm{T}$ lymphocytes by binding to $\mathrm{PD}-1$ and reduce the activity of $\mathrm{T}$ lymphocytes, thereby negatively regulating the antitumor immune response [33, 34]. Although the clinical application of PD-1/PD-L1 inhibitors is limited in $\mathrm{CRC}$, the role of $\mathrm{PD}-\mathrm{L} 1$ in promoting progression and immune evasion of CRC could not be ignored [35]. Downregulation of PD-L1 expression or blocking PD-L1 signals could block progression and immune evasion of CRC. For instance, PPAR $\gamma$ agonists could increase the expression of PD-L1 at transcriptional and protein levels in CRC cell lines and induce immune evasion [36]. Given the complexity and diversity of tumor cell gene expression regulation, it is indispensable to further investigate the molecular mechanisms underlying $\mathrm{PD}-\mathrm{L} 1$ regulation and find new intervention targets.

In this research, we reported that EMT was essential for PD-L1 expression. Based on the bioinformatics analysis, we found that the EMT score was positively related to the expression of the most significant immunomodulators. EMT score was also positively related to increased infiltration of TIICs and hyperactive cancer immunity cycles. In addition, we revealed that a high EMT score was positively correlated with most immune checkpoints expression and negatively correlated with Tumor Purity. Previous research indicated that ZEB1 was the critical regulator for PD-L1 expression among a panel of transcription factors in the EMT process in breast cancer [24]. Similarly, we validated that ZEB1 positively regulated $\mathrm{PD}-\mathrm{L} 1$ expression in $\mathrm{CRC}$. In addition, the positive correlation between ZEB1 and PD-L1 in clinical samples was also observed.
In the past few decades, an increasing number of traditional Chinese medicines have been reported to exert tumor-suppressive functions, which may open up novel insights for tumor therapy $[37,38]$. Biochanin $\mathrm{A}$ is an oxymethylated isoflavone compound, which is widely present in some edible plants. Existing studies have shown that Biochanin A has a variety of pharmacological effects, such as antitumor, anti-inflammatory, antibacterial, hypoglycemic, antioxidant, and neuroprotection [39]. Biochanin A could induce $S$ phase arrest and apoptosis of lung cancer cells [40]. In addition, Biochanin A negatively regulated the proliferation and migration of lung cancer cells by inhibiting the VEGF/VEGFR2 signaling pathway [41]. Furthermore, the antitumor effect of Biochanin A was also confirmed in pharyngeal squamous cell carcinoma [42] and breast cancer [43]. All the evidence suggested that Biochanin A was a broad-spectrum antitumor candidate. In this research, we found that Biochanin A significantly downregulated ZEB1 expression and blocked the EMT process. Moreover, Biochanin A inhibited proliferation, migration, and invasion of HCT116 and SW620 cells, which could be rescued by ZEB1 overexpression. Given the critical role of ZEB1 in regulating $\mathrm{PD}$-L1 expression, we speculated that Biochanin A may downregulate PD-L1 via inhibiting ZEB1 expression. Notably, Biochanin A indeed suppressed PD-L1 expression, which was rescued by ZEB1 overexpression.

\section{Conclusion}

In summary, we reported that the EMT process is related to features of TME in CRC in this research. Besides, ZEB1 is a crucial regulator for $\mathrm{PD}-\mathrm{L} 1$ expression, and pharmacological inhibition of ZEB1 using Biochanin A downregulated PD-L1 in vivo. Overall, we uncovered that Biochanin $A$ is a promising antitumor candidate by negatively regulating the EMT process and PD-L1 expression.

\section{Data Availability}

The data of this research are available from the corresponding author upon request.

\section{Conflicts of Interest}

The authors declare that they have no conflicts of interest.

\section{Authors' Contributions}

Dong Hua and Junying $\mathrm{Xu}$ designed the study and participated in coordination and project control. Junying $\mathrm{Xu}$, Xuejing Yang, and Jie Mei collected the public data and conducted the bioinformatics analysis. Junying $\mathrm{Xu}$, Xuejing Yang, Jiadong Pan, Honghong Fan, and Jie Mei performed in vitro and in vivo assays. Junying $\mathrm{Xu}$ and Xuejing Yang wrote the draft. Dong Hua revised the manuscript. Dong Hua and Junying $\mathrm{Xu}$ got financial support. All authors reviewed and approved the final edition. Junying $\mathrm{Xu}$, Xuejing Yang, and Jiadong Pan contributed equally to this research. 


\section{Acknowledgments}

This work was supported by the Top Medical Expert Team of the 2021 Taihu Talent Plan Project to Dong Hua and the Wuxi Science and Technology Bureau Fund (no. N20202018) to Junying Xu.

\section{Supplementary Materials}

Figure S1: Correlations between EMT score and scores estimated by ESTIMATE algorithm. (a) Positive correlation between EMT score and immune score. (b) Positive correlation between EMT score and ESTIMATE Score. (c) Positive correlation between EMT score and Stromal Score. (d) Negative correlation between EMT score and Tumor Purity. Figure S2: The efficiency of ZEB1 silencing and overexpression in CRC cells. (a, b) The efficiency of ZEB1 silencing in CRC cells was assessed by qRT-PCR and Western blotting. (c, d) The efficiency of ZEB1 overexpression in CRC cells was assessed by qRT-PCR and Western blotting. (Supplementary Materials).

\section{References}

[1] R. L. Siegel, K. D. Miller, H. E. Fuchs, and A. Jemal, "Cancer statistics, 2021," CA: A Cancer Journal for Clinicians, vol. 71, no. 1, pp. 7-33, 2021.

[2] K. Burgers, C. Moore, and L. Bednash, "Care of the colorectal cancer survivor," American Family Physician, vol. 97, no. 5, pp. 331-336, 2018.

[3] F. Liu, X.-L. Xiao, Y.-J. Liu et al., "CircRNA_0084927 promotes colorectal cancer progression by regulating miRNA20b-3p/glutathione S-transferase mu 5 axis," World Journal of Gastroenterology, vol. 27, no. 36, pp. 6064-6078, 2021.

[4] C. Lachat, P. Peixoto, and E. Hervouet, "Epithelial to mesenchymal transition history: from embryonic development to cancers," Biomolecules, vol. 11, no. 6, 2021.

[5] K. Behzatoglu, "Osteoclasts in tumor biology: metastasis and epithelial-mesenchymal-myeloid transition," Pathology and Oncology Research, vol. 27, Article ID 609472, 2021.

[6] L. Wang, A. Saci, P. M. Szabo et al., "EMT- and stroma-related gene expression and resistance to $\mathrm{PD}-1$ blockade in urothelial cancer," Nature Communications, vol. 9, no. 1, 3503 pages, 2018.

[7] A. R. Jung, C.-H. Jung, J. K. Noh, Y. C. Lee, and Y.-G. Eun, "Epithelial-mesenchymal transition gene signature is associated with prognosis and tumor microenvironment in head and neck squamous cell carcinoma," Scientific Reports, vol. 10, no. 1, 3652 pages, 2020.

[8] S. Brabletz, H. Schuhwerk, T. Brabletz, and M. P. Stemmler, "Dynamic EMT: a multi-tool for tumor progression," The EMBO Journal, vol. 40, no. 18, Article ID e108647, 2021.

[9] Q. Hua, Z. Sun, Y. Liu et al., "KLK8 promotes the proliferation and metastasis of colorectal cancer via the activation of EMT associated with PAR1," Cell Death \& Disease, vol. 12, no. 10, 860 pages, 2021.

[10] L. Xu, C. Deng, B. Pang et al., "TIP: a web server for resolving tumor immunophenotype profiling," Cancer Research, vol. 78, no. 23, pp. 6575-6580, 2018.

[11] A. Liberzon, C. Birger, H. Thorvaldsdóttir, M. Ghandi, J. P. Mesirov, and P. Tamayo, "The molecular signatures Database hallmark gene set collection," Cell systems, vol. 1, no. 6, pp. 417-425, 2015.
[12] P. Charoentong, F. Finotello, M. Angelova et al., "Pan-cancer immunogenomic analyses reveal genotype-immunophenotype relationships and predictors of response to checkpoint blockade," Cell Reports, vol. 18, no. 1, pp. 248-262, 2017.

[13] K. Yoshihara, M. Shahmoradgoli, E. Martínez et al., "Inferring tumour purity and stromal and immune cell admixture from expression data," Nature Communications, vol. 4, no. 1, 2612 pages, 2013.

[14] T. Li, J. Fu, Z. Zeng et al., "TIMER2.0 for analysis of tumorinfiltrating immune cells," Nucleic Acids Research, vol. 48, no. W1, pp. W509-W514, 2020.

[15] J. Racle, K. de Jonge, P. Baumgaertner, D. E. Speiser, and D. Gfeller, "Simultaneous enumeration of cancer and immune cell types from bulk tumor gene expression data," Elife, vol. 6, 2017.

[16] E. Becht, N. A. Giraldo, L. Lacroix et al., "Estimating the population abundance of tissue-infiltrating immune and stromal cell populations using gene expression," Genome Biology, vol. 17, no. 1, p. 218, 2016.

[17] F. Finotello, C. Mayer, C. Plattner et al., "Molecular and pharmacological modulators of the tumor immune contexture revealed by deconvolution of RNA-seq data," Genome Medicine, vol. 11, no. 1, p. 34, 2019.

[18] B. Ru, C. N. Wong, Y. Tong et al., "TISIDB: an integrated repository portal for tumor-immune system interactions," Bioinformatics, vol. 35, no. 20, pp. 4200-4202, 2019.

[19] J. Mei, T. Yan, Y. Huang et al., “A DAAM1 3'-UTR SNP mutation regulates breast cancer metastasis through affecting miR-208a-5p-DAAM1-RhoA axis," Cancer Cell International, vol. 19, no. 1, p. 55, 2019.

[20] J. Mei, Y. Liu, X. Yu et al., "YWHAZ interacts with DAAM1 to promote cell migration in breast cancer," Cell Death Discovery, vol. 7, no. 1, p. 221, 2021.

[21] M. Xu, Y. Han, G. Liu et al., "Preclinical study of a fully human anti-PD-L1 antibody as a theranostic agent for cancer immunotherapy," Molecular Pharmaceutics, vol. 15, no. 10, pp. 4426-4433, 2018.

[22] C. Li, H. Chi, S. Deng et al., "THADA drives Golgi residency and upregulation of PD-L1 in cancer cells and provides promising target for immunotherapy," Journal for immunotherapy of cancer, vol. 9, no. 8, 2021.

[23] L. Chen, D. L. Gibbons, S. Goswami et al., "Metastasis is regulated via microRNA-200/ZEB1 axis control of tumour cell PD-L1 expression and intratumoral immunosuppression," Nature Communications, vol. 5, no. 1, 5241 pages, 2014.

[24] M. Z. Noman, B. Janji, A. Abdou et al., "The immune checkpoint ligand PD-L1 is upregulated in EMT-activated human breast cancer cells by a mechanism involving ZEB-1 and miR-200," OncoImmunology, vol. 6, no. 1, e1263412 pages, 2017.

[25] Y. Liang, Y. Liu, Q. Zhang, H. Zhang, and J. Du, “Tumorderived extracellular vesicles containing microRNA-1290 promote immune escape of cancer cells through the Grhl2/ ZEB1/PD-L1 axis in gastric cancer," Translational Research, vol. 231, pp. 102-112, 2021.

[26] L. Zhao, Y. Liu, J. Zhang, Y. Liu, and Q. Qi, "LncRNA SNHG14/miR-5590-3p/ZEB1 positive feedback loop promoted diffuse large $\mathrm{B}$ cell lymphoma progression and immune evasion through regulating PD-1/PD-L1 checkpoint," Cell Death \& Disease, vol. 10, no. 10, 731 pages, 2019.

[27] Y. Wang, J. J. Li, and Y. M. Chen, "Biochanin A extirpates the epithelial-mesenchymal transition in a human lung cancer," Experimental and Therapeutic Medicine, vol. 15, no. 3, pp. 2830-2836, 2018. 
[28] A. Anichini, V. E. Perotti, F. Sgambelluri, and R. Mortarini, "Immune escape mechanisms in non small cell lung cancer," Cancers, vol. 12, no. 12, 2020.

[29] C. Robert, J. Schachter, G. V. Long et al., "Pembrolizumab versus ipilimumab in advanced melanoma," New England Journal of Medicine, vol. 372, no. 26, pp. 2521-2532, 2015.

[30] R. S. Herbst, P. Baas, D.-W. Kim et al., "Pembrolizumab versus docetaxel for previously treated, PD-L1-positive, advanced non-small-cell lung cancer (KEYNOTE-010): a randomised controlled trial," The Lancet, vol. 387, no. 10027, pp. 1540-1550, 2016.

[31] R. Nanda, L. Q. M. Chow, E. C. Dees et al., "Pembrolizumab in patients with advanced triple-negative breast cancer: phase ib KEYNOTE-012 study," Journal of Clinical Oncology, vol. 34, no. 21, pp. 2460-2467, 2016.

[32] P. Armand, M. A. Shipp, V. Ribrag et al., "Programmed death1 blockade with pembrolizumab in patients with classical hodgkin lymphoma after brentuximab vedotin failure," Journal of Clinical Oncology, vol. 34, no. 31, pp. 3733-3739, 2016.

[33] W. Zou, J. D. Wolchok, and L. Chen, "PD-L1 (B7-H1) and PD-1 pathway blockade for cancer therapy: mechanisms, response biomarkers, and combinations," Science Translational Medicine, vol. 8, no. 328, 328rv4 pages, 2016.

[34] Y. Wang, H. Wang, H. Yao, C. Li, J.-Y. Fang, and J. Xu, "Regulation of PD-L1: emerging routes for targeting tumor immune evasion," Frontiers in Pharmacology, vol. 9536 pages, 2018.

[35] P. Agarwal, D. T. Le, and P. M. Boland, "Immunotherapy in colorectal cancer," Advances in Cancer Research, vol. 151, pp. 137-196, 2021.

[36] T. Gutting, V. Hauber, J. Pahl et al., "PPAR $\gamma$ induces PD-L1 expression in MSS+ colorectal cancer cells," OncoImmunology, vol. 10, no. 1, Article ID 1906500, 2021.

[37] O. R. Oyenihi, A. B. Oyenihi, J. O. Erhabor, M. G. Matsabisa, and O. O. Oguntibeju, "Unravelling the anticancer mechanisms of traditional herbal medicines with metabolomics," Molecules, vol. 26, no. 21, 2021.

[38] Y. Wang, X. Zhang, Y. Wang et al., "Application of immune checkpoint targets in the anti-tumor novel drugs and traditional Chinese medicine development," Acta Pharmaceutica Sinica B, vol. 11, no. 10, pp. 2957-2972, 2021.

[39] A. Sarfraz, M. Javeed, M. A. Shah et al., "Biochanin A: a novel bioactive multifunctional compound from nature," The Science of the Total Environment, vol. 722, Article ID 137907, 2020.

[40] Y. Li, H. Yu, F. Han, M. Wang, Y. Luo, and X. Guo, "Biochanin A induces S phase Arrest and apoptosis in lung cancer cells," BioMed Research International, vol. 2018, Article ID 3545376, 2018.

[41] X. Lai, Y. Li, and M. Gao, "Biochanin A regulates the growth and migration of NSCLC through suppressing the VEGF/ VEGFR2 signaling pathway," Oncology Research Featuring Preclinical and Clinical Cancer Therapeutics, vol. 2018, 2018.

[42] I.-A. Cho, S.-J. You, K.-R. Kang et al., "Biochanin-A induces apoptosis and suppresses migration in $\mathrm{FaDu}$ human pharynx squamous carcinoma cells," Oncology Reports, vol. 38, no. 5, pp. 2985-2992, 2017.

[43] G. Ren, Z. Shi, C. Teng, and Y. Yao, "Antiproliferative activity of combined Biochanin A and ginsenoside $\mathrm{Rh}(2)$ on MDAMB-231 and MCF-7 human breast cancer cells," Molecules, vol. 23 , no. 11, 2018. 